By a generalization of the idea of chain folding first derived from the X-ray study of the fibrous proteins, we arrive at the concept of the corpuscular proteins, and then at the interpretation of denaturation as a process of disorganization whereby the peptide chains are liberated from their specific configurations ; and it has in fact been demonstrated conclusively by $\mathrm{X}$-ray methods (leading to actual industrial processes) that artificial fibrous proteins can be spun, after denaturation, from many corpuscular proteins. By an extension of the idea of close packing of the side-chains, we arrive at the concept of the protein lamina, probably again usually with all or most of the polars on one side and the nonpolars on the other ; and the next step is the building of combinations of two or more laminæ, sometimes alone and sometimes sandwiched with accessory groupings, such as lipoid laminæ. It was an early deduction (1936) from X-ray fibre and monolayer data that the egg albumin molecule consists probably of four superposed laminxe of folded polypeptide chains, and recent physico-chemical results by Lundgren and Palmer strongly support that suggestion. Also, the X-ray findings of Perutz and Boyes-Watson now point to a similar conclusion for the structure of the hæmoglobin molecule.

The full pattern along the molecular chains in both the keratin-myosin-fibrinogen group and the collagen group is on a giant scale as molecules go, so great as to fall within the range of the electron microscope, as has been shown by the recent very beautiful experiments of F. O. Schmitt and his team. Even in unstained collagen fibrils, they have demonstrated a system of light and dark bands corresponding to the $640 \mathrm{~A}$. X-ray fibre period found independently by Bear and Kratky, while in fibrils from certain clam muscles, after treatment with phospho-tungstic acid, they have succeeded in revealing more detail still-a most intriguing arrangement of stainable regions forming a systematically distorted hexagonal net. The impression one gets from combined X-ray and electron microscope studies is of 'patterns within patterns', of successive levels of organization proceeding from the basic plans revealed in large-angle X-ray photographs up to the relatively enormous fibrils, and thence to combinations of fibrils. In this progressive building. up process and the transition from one level to the next, we possibly have to do with small proportions of non-protein components, for example, nucleotides and carbohydrates ; and the suggestion is that it is now necessary to estimate such small-proportion components with much greater precision, to see if they are indeed in definite and structurally significant stoichiometric rolation to the remainder.

The X-ray study of the polynucleotides, like the chemistry of these all-important structures, is as yet practically in its infancy. An encouraging start has been made with sodium thymonucleate, the diffraction pattern of which indicates a columnar piling of flattish nucleotides spaced at almost exactly the same distance as the separation of successive side-chains in an extended polypeptide, and similar features are suggested by preliminary photographs of nucleic acids of the ribose type; but otherwise all this great world remains to be conquered. The proffered reward is tremendous, for, whatever else is in doubt, it seems clear enough now that the innermost secrets of biosynthesis rest above all with the proteins and the nucleic acids.

\section{X-RAY DIFFRACTION IN INORGANIC CHEMISTRY, METALLURGY AND MINERALOGY}

\author{
By DR. H. LIPSON*
}

\section{Chemistry of the Solid State}

$\mathrm{T}$ HE discovery by Friedrich, Knipping and Laue of the diffraction of X-rays by crystals opened up now vistas to those interested in the structure of matter. It was now possible to determine the environment of individual atoms, and this inevitably led to a new and lively interest in the properties of matter, the further study of which had seemed to be opposed by an impassable barrier. Moreover, a new status was given to crystallography. No longer was it to be regarded as a subject of limited interest; all men of science had to have a sound knowledge of crystallographic principles and the ability to apply them to their special problems.

Inorganic chemistry, in particular, was revolutionized by the new concepts that emerged from the determinations of the first crystal structures. Rock salt-the first crystal to be analysed-provided a striking example. It was known, of course, that it dissociates into ions of sodium and chlorine when it dissolves in water; but it was supposed that in the solid state the ions were associated in pairs to form molecules of $\mathrm{NaCl}$; this was the readiest way of accounting for the equality of numbers of sodium and chlorine atoms. Barlow, in 1883, had suggested the true crystal structure; but as his suggestion was not supported by direct experimental evidence it had not reached the general consciousness of chemists. X-ray diffraction showed that Barlow's structure was correct and that there were therefore no molecules of $\mathrm{NaCl}$, but only regular arrangements of chlorine and sodium atoms in which each atom was surrounded by six of the other sort. The equality of numbers of the atoms then appeared to be a result of the equality of their chemical valencies. In this way the beginnings of a new chemistry-crystal chemistry-arose.

This subject may be called the chemistry of the solid state as distinct from the chemistry of the liquid state, which was, and still is, the chemist's main interest. As methods for determining crystal structures improved, so more and more chemical compounds were analysed and provided data for the theoretical chemist. Not only was it possible to determine bond-lengths and inter-bond angles, but also to study ions and infinite complexes that could not exist in the liquid state.

Hydrated inorganic compounds provide a good example of the fruits of this study. The structures of salts with four or six molecules of water could be adequately explained on the basis of Werner's co-ordination theory, the water molecules forming simple regular arrangements about the cation; but what of compounds such as $3 \mathrm{CdSO}_{4} \cdot 8 \mathrm{H}_{2} \mathrm{O}$ or $\mathrm{H}_{3} \mathrm{PW}_{12} \mathrm{O}_{40} .29 \mathrm{H}_{2} \mathrm{O}$ ? The determination of the structures of compounds such as these showed that, in addition to providing a regular grouping around the cation, the water molecules because of their polar

* A review based upon papers read by Dr. A. F. Wells, Dr. H. Iipson and Dr. W. H. Taylor at a meeting held in the Royal Institution on November 10 to celebrate the fiftieth a nniversary of the discovery of X-rays. 
nature could help to provide links in the chains of forces holding the crystal together.

This indeed is one of the rather surprising results that have emerged from crystal analysis-the large part that geometry plays in influencing atomic arrangements. Apparently water molecules can take up their places in a crystal because they can adapt themselves to the space available, and the structure has then a lower free energy than if an arrangement of anions and cations was built up without them. Similarly, molecular compounds such as $\mathrm{CH}_{8} \mathrm{I}_{3} 3 \mathrm{~S}_{8}$ exist because their molecules are so shaped that they fit well together.

Nevertheless, the importance of crystal chemistry lay not in the geometry of structures but in the forces holding the structures together. When the main structure types were analysed, the process of systematization and classification began, and in its general outlines the scheme is now fairly clear. The forces that hold the atoms together in crystals such as rock salt were clearly not the only forces available, for these cannot exist in crystals, such as those of the elements, in which there are atoms of only one kind. Thus the theory of the covalent bond was developed; a pair of atoms was supposed to have a lower energy if an electron was shared between them, this reduction of energy being equivalent to a force between the atoms. Clearly, this type of bond could suffice for many different crystals: more than one electron could be shared between a pair of atoms, or, on the other hand, an electron could be shared between many atoms. This latter case found its limiting expression in the metallic state, in which each available. electron is shared between all the atoms in a crystal. The only type of bond that was not included is the van der Waals bond, which is a result of the ordinary gravitational attraction and is much weaker than the other types of bond.

The main outlines of erystal chemistry are based on these four types of force. But as happens so often in science, these main outlines are not enough; a close examination of structural details showed that effective atomic radii did not fall into the various groups in as orderly a fashion as was expected. It is now realized that bonds in general do not fall precisely into any one class; any particular bond then may have both an electrovalent and covalent character : there is no clear-cut division. This is the present state of the subject. Advances will be made as more data become available, and the relations between bond-length and type of interatomic force become accurately established. But such data must be accurate; some laboratories produce work of high accuracy, and a general levelling up of the standards of all to that of the best would be desirable.

\section{The Metallic State}

In the study of the metallic state, progress has been slower, for the raw material of the crystallographer-the single crystal-was not readily available. However, the discovery by Hull, and by Debye and Schorrer, that even powdered crystals could give interpretable X-ray diffraction photographs, brought the ordinary polycrystalline metal within the scope of X-ray analysis. The powder method and its modifications have been used with great advantage to deal with many problems, both industrial and academic.

The determination of equilibrium diagrams pro- vides perhaps the most important example of the application of $\mathrm{X}$-ray methods. Metallurgists used various methods, each with its particular advantages and disadvantages; but X-ray powder photographs provided much clearer characteristics than any other method for the identification of phases. Particularly noteworthy was the pioneer work of Westgren, and outstanding among Westgren's work was his identification of the high-temperature phases in pure iron; these results could not have been obtained by other methods. All this, however, does not mean that the $\mathrm{X}$-ray diffraction method has displaced other methods. For certain purposes X-ray methods have not been so successful as was originally hoped; and the general opinion at present is that the $\mathrm{X}$-ray worker should, if possible, supplement his results by those obtained with other methods.

An unexpected use of X-ray diffraction is the measurement of internal stresses in metals. Elastic changes in a piece of metal are accompanied by corresponding changes in the lattice of each crystal, and the lattice changes can be measured by means of X-rays. It is perhaps too early to give a considered opinion about the ultimate value of this use of X-rays : at present it seems promising, but it should be interesting to see in about five years time whether it still has a place in the industrial armoury, or whether it has been displaced by methods simpler and more easily applied.

Of more undisputed utility is the detection of preferred orientation in substances due to the method of fabrication. For example, when metal is rolled into sheet form, the crystal grains tend to align themselves with similar orientations, and the resultant materials may have properties approximating to those of a single crystal. This is often undesirable, and the fact that the preferred orientation can be detected by $\mathrm{X}$-rays before the material is put into use makes it possible to control the phenomenon satisfactorily.

The fundamental importance of X-ray diffraction, however, lies in the insight it gives into atomic arrangements. The work of Bradley, first in the determination of the $\gamma$-structure and later in the determination of the structure of the orthorhombic phase $\mathrm{NiAl}_{3}$, has been outstanding in this connexion. The determination of the crystal structures of the various phases that appear in equilibrium diagrams is a necessary preliminary to the complete understanding of these diagrams. Ideally, we should be able to calculate the free energy of any atomic arrangement. Far as we are from this ideal, a start has been made; in some instances it is possible to calculate how the free energies of the valency electrons (which characterize the metallic state) vary with the composition of the alloy, and these free energies often decide which phases will be stable. The results, beautifully expressed in the form of the Brillouinzones theory, agree well with the empirically deduced Hume-Rothery rules, which relate the stable structure to the number of valency electrons associated with each atom. This is the first successful attempt to correlate theoretically the existence of a phase and its crystal structure.

All this is concerned with what we might call the 'statics' of metals ; within the last few years a new type of study, which we might call 'kinetics', has begun, and in the future it will probably overshadow in importance the earlier work. Few alloys in common use are in equilibrium states; transition states with enhanced strength or hardness are usually sought 
for. For example, duralumin-ideally an alloy of 4 per cent of copper in aluminium-has a Brinnell hardness number of about 60 if the copper is all in solid solution; but if it is left for a few days at room temperature the copper tends to precipitate and the hardness number increases to about 90 . If singlecrystal photographs are taken it is found that streaks appear as the hardening develops, and Preston and Guinier have both ascribed these streaks to the presence of sheets of copper atoms parallel to the (100) planes. Preston has carried the investigation a stage further; he annealed a single crystal at $200^{\circ} \mathrm{C}$. and found that the streaks developed maxima and finally broke up into definite spots. These he found to belong to a structure of the $\mathrm{CaF}_{2}$ type. Now the stable phase $\theta$ has not this structure, and Preston ascribed this precipitation of a metastable phase to the fact that it had a plane of atoms in common with the aluminium structure. In this way the increase in surface energy that occurs when a phase is precipitated is largely eliminated and the whole system has a lower free energy than a system composed of stable structures on the same scale.

Another example of the study of the kinetics of metals is provided by the alloy $\mathrm{Cu}_{4} \mathrm{FeNi}_{3}$. This alloy is face-centred cubic at temperatures above about $800^{\circ} \mathrm{C}$. but at lower temperatures breaks up into two face-centred cubic phases. Daniel and Lipson have shown that in the process of dissociation the alloy gives a powder photograph that has diffuse bands on either side of the main diffraction lines. The bands can be explained as 'ghosts' due to a periodic deformation of the lattice on a scale of 100-1,000 A. The intensities and positions of these 'ghosts' change as the alloy is given different heat treatments, and from measurements of these quan tities it is possible to obtain valuable information about the process of dissociation.

\section{Structure of Minerals}

Similar problems have arisen in the study of silicates. Many interesting types of diffraction effects have been observed and these should throw a great deal of light on the mechanism of isomorphous replacement. Before dealing with these effects, however, it is necessary to outline the types of structure that occur and to show the part that $\mathrm{X}$-ray analysis has played in their classification.

Before the advent of X-ray analysis, mineralogists had classified silicates according to their physical and chemical properties. On the basis of the physical properties, the classification was mainly correct; on the basis of their chemistry it was quite wrong. To explain the different proportions of silicon and oxygen in the different compounds a series of 'silicic acids' was postulated. The researches by W. L. Bragg's school in Manchester showed that the underlying principles were in reality much simpler; oach silicon atom is always surrounded by oxygen atoms at the corners of a regular tetrahedron, and the oxygen atoms are sometimes shared between two tetrahedra. By varying the number of tetrahedra that share oxygen atoms and the number of oxygen atoms in one tetrahedron that are shared, a great variety of silicon-oxygen ratios can be produced.

Once this principle was established, the rest of the structural details fell naturally into place. The system of linked $\mathrm{SiO}_{4}$ tetrahedra in a crystal behaves as an anion, and its negative charge is balanced by the positive charges on metallic cations. The whole structure obeys Pauling's rules, which in effect postulate that there must be no accumulation of charge on any small group of atoms. These rules are essential to a proper understanding of silicate structures and of the extensive isomorphous replacement that is found in naturally occurring minerals. In this replacement the most important factor is that the replacing atom should be similar in size to that which is replaced; the number of replacing atoms will then be governed by their valencies, in accordance with Pauling's rules. This is another example of the importance of geometrical considerations; the chemical nature of the atoms is of negligible influence.

Isomorphous replacement does not always take place in a simple way, and some mixed crystals produce the interesting diffraction effects referred to earlier. The felspars, for example, have been studied in detail by W. H. Taylor and his school. These minerals have a framework of tetrahedra in which all the oxygen atoms are shared between neighbouring tetrahedra. Considerable variation is possible in the dimensions of this framework, and so cations of different sizes can be accommodated. Difficulties arise, however, when two different atoms have to be accommodated in the same structure, as happens in the potash soda felspars. It potassium predominates, the structure is an ordinary solid solution; but if a large amount of sodium is present an interesting type of structure may occur. In these perthitic structures, lamellæ which are successively potassium-rich and sodium-rich are produced; in the former the framework is extended, and in the latter contracted. This variation in structure occurs in a 'single' crystal, and the diffraction effects can therefore be studied in some detail. The structure is, however, rather complicated and a complete explanation of all the effects observed has not yet been obtained. It is certain that many interesting problems of this type will occur in work on minerals.

Another kind of defect occurs in the mineral chrysotile, the most important raw material of the asbestos industry. The X-ray photograph contains a mixture of sharp reflexions and reflexions with 'tails' spread out along the layer lines. The problem of the determination of the defect that gives this diffraction pattern has been partially solved by Warren and by Aruja, but publication of the complete solution is still awaited.

There are thus still many interesting problems awaiting solution in the field of $\mathrm{X}$-ray diffraction, and it is in the realm of the distorted or imperfect crystal that the various branches of the subject are likely to link together. For a successful study of these phenomena the technique of taking $\mathbf{X}$-ray photographs must be advanced; many of the phenomena observed nowadays would scarcely have been noticed with the apparatus used, say, twenty years ago. Hightemperature cameras and cameras of large radius will have to be available, and crystal-reflected radiation will have to be used. In addition, X-ray tubes of greatly increased power will be necessary so that exposures will not be unduly prolonged and so that finer collimators can be used. Only then will these problems of the secondary X-ray diffraction effects-which may lead into quite unexpected regions-be attacked and solved on the scale they require. 